\title{
Thick and widened high quality heavily boron doped diamond single crystals synthetized with high oxygen flow under high microwave power regime
}

\author{
R. Issaoui ${ }^{\mathrm{a}, *}$, J. Achard $^{\mathrm{a}}$, L. William ${ }^{\mathrm{a}}$, L. Mehmel $^{\mathrm{a}}$, M.-A. Pinault-Thaury ${ }^{\mathrm{b}}$, F. Bénédic ${ }^{\mathrm{a}}$ \\ ${ }^{a}$ LSPM-CNRS, LABORATOIRE des Sciences des Procédés et des MATERIAUX, Université \\ PARIS 13, Sorbonne PARIS Cité, 99 AVENUE JEAN BAPTISTE Clément, 93430 \\ VILLETANEUSE, FRANCE \\ ${ }^{b}$ Groupe d'Etude de LA MATIERE Condensée (GEMAC-UMR8635), CNRS, Université de \\ VERSAILLES St-Quentin-En-Yvelines (UVSQ), Université PARIS-SACLAY, 45 AV des ETATS \\ Unis, 78035 VERSAILLES Cedex, FRANCE
}

*Corresponding author: riadh.issaoui@1spm.cnrs.fr

\begin{abstract}
The aim of this paper is to optimize the growth conditions of thick boron doped diamond single crystals, which requires the use of high microware power density to have high growth rate, in order to allow enlarging CVD deposited layers keeping both high boron doping level and high crystal quality. It is shown that the use of a high amount of oxygen (1 to $2 \%)$ in the gas phase leads to $1 \mathrm{~mm}$ lateral growth after the growth of $500 \mu \mathrm{m}$ thick CVD diamond layer. Thus, the surface is increased from $10 \mathrm{~mm}^{2}$ to $18 \mathrm{~mm}^{2}$. The obtained films exhibit high crystal quality confirmed by Raman spectroscopy and confocal microscopy. Depending on the gas composition introduced in the reactor, the boron concentration varied between $5 \times 10^{19} \mathrm{at} / \mathrm{cm}^{3}$ and $3 \times 10^{20} \mathrm{at} / \mathrm{cm}^{3}$ as measured by SIMS.
\end{abstract}

\section{Introduction}

Diamond is known to be one of the most suitable wide band gap semiconductor for high power devices [1]. Microwave Plasma Assisted CVD (MPACVD) is an appropriate method to have a good control of boron-doped diamond growth for electronic devices [2,3], even if it is still difficult to reach heavily boron doping when thick layers are de- sired. Indeed, previous studies have shown soot formation within the plasma when both high amounts of boron precursors and high micro- wave power density are used [4]. A compromise using medium power/ pressure (2400 W/140 mbar) coupled to the addition of a very small amount of oxygen in the gas phase $(0.25 \%)$ has already been determined for the growth of thick and highly boron doped single crystal diamond (few hundreds of micrometers doped at 1020 at $/ \mathrm{cm} 3$ ) [4-7]. Nevertheless, such a compromise of microwave power density leads to a low growth rate and some crystal defects can be formed during the growth. Moreover, the surface area tends to decrease that is a very limiting point for the growth of thick films. Indeed, the development of high-power vertical devices based on diamond relies on the development of large and thick 
heavily boron doped layers $[8,9]$. Another point is that these films should have a high quality and a limited dislocations density. This is a very crucial point because several growth resuming of active layers are required to fabricate devices such as vertical Schottky diode. The purpose of this work is to develop a new protocol allowing coupling high microwave power density and high boron doping to reach high quality thick boron doped grown layers with an enlargement of the top surface.

\section{Experimental setup}

(100)-oriented HPHT (High Pressure - High Temperature) diamond substrates were used to grow several samples with different deposition conditions, the aim being to evaluate their impact in terms of boron concentration and structural quality. The edges of the substrates are oriented (100) in order to avoid induced stress because of the top surface and edge orientation. The dimensions for all substrates are quite similar $\left(1.5 \mathrm{~mm} \pm 5 \mu \mathrm{m}\right.$ height and $3.15 \times 3.15 \mathrm{~mm}^{2}$ large, the deviation for lateral dimensions is $\pm 3 \mu \mathrm{m}$ ). Before the growth, all samples were acid and plasma treated as described elsewhere [10]. In order to improve our growth conditions, the microwave power/pressure couple was increased in comparison with previous results $[4,11,12]$, from $140 \mathrm{mbar} / 2400 \mathrm{~W}$ to $170 \mathrm{mbar} / 2700 \mathrm{~W}$. The impact of oxygen on such a process has been also investigated by increasing its concentration from $0.25 \%$ to $2 \%$ in the gas phase. The methane was increased at the same time from $5 \%$ to $10 \%$ to compensate the surface etching due to the increase of oxygen flow [13], the aim being to maintain an acceptable growth rate for the growth of thick film. Diborane was used as boron precursor. Three ratios of $(\mathrm{B} / \mathrm{C})_{\mathrm{gas}}, 15,000$, 20,000 and 30,000 ppm, were used in order to increase the boron doping level. Six (6) separated samples were deposited at $920{ }^{\circ} \mathrm{C}$ and $200 \mathrm{sccm}$ of total gas flow. The temperature was measured by a Williamson bichromatic pyrometer. The grown film thicknesses were obtained by measuring the mass gain after growth using a balance (METTLER TOLEDO XPE 205 DeltaRange) and confirmed by measuring the height difference before and after the growth with a caliper (Mitutuyo). The surface morphology was observed using a laser confocal microscope (Keyence VK 9710). Raman spectroscopy (Jobin-Yvon LabRam HR800) was carried out at room temperature using a $473 \mathrm{~nm}$ laser to evaluate the crystalline quality of the samples. The boron concentration [B] was measured by secondary ion mass spectrometry (SIMS) in a CAMECA IMS7f equipment using a Cs+ ion beam. Profiles were performed in the 2-3 $\mu \mathrm{m}$ top layer. The quantification of the boron concentration was made thanks to an ion-implanted diamond standard with a known dose of boron. This method typically gives the boron concentration of the epilayers with a $10 \%$ relative uncertainty. The summary of growth conditions is given in Table 1 .

\section{Results and discussion}

Fig.1 shows the evolution of the growth rate with the oxygen and methane content for a constant $(\mathrm{B} / \mathrm{C})_{\text {gas }}$ equal to $15,000 \mathrm{ppm}$. As expected, this parameter increases when both methane and oxygen concentrations increase, showing that the etching effect related to oxygen can be compensated by increasing carbon-containing species in the plasma. Thus, a growth rate as high as $4 \mu \mathrm{m} \cdot \mathrm{h}^{-1}$ is reached with a methane concentration of $10 \%$ coupled with $2 \%$ of oxygen. Note that sample D has been grown during $130 \mathrm{~h}$ without any soot formation as it has been 
already demonstrated for intrinsic diamond [12]. This demonstrates that the use of higher oxygen concentrations allows diamond deposition with a high diborane concentration at high microwave power for a very long synthesis time.

After the growth, the films deposited with various methane and oxygen ratios for a given $(\mathrm{B} / \mathrm{C})_{\text {gas }}$ of $15,000 \mathrm{ppm}$ were observed by laser confocal microscope and the corresponding surface morphologies are presented in Fig.2. No epitaxial crystallites are visible on the surface of the samples whatever the growth conditions used.

Raman spectroscopy analysis was also performed on these layers, showing similar spectra. The Raman shift spectra are presented in Fig.3a showing that no graphitic phase is observed. The Fano effect is clearly observed on the diamond peak as well as 2 peaks around 600 and $1100 \mathrm{~cm}^{-1}$ which is typical of diamond films with a boron doping level close to or higher than $5 \times 1019$ at $/ \mathrm{cm} 3[14,15]$. Fig.3b shows the Raman spectra of the samples focused on the day diamond peak. The diamond peak is around $1330 \mathrm{~cm}-1$ and its width at half maximum (FWHM) variates from 4 to 5.8 when the oxygen was increased from 0.25 up to $2 \% \mathrm{~cm}-1$ as reported in Table 2 for a similar thickness. This result is consistent with the expected doping level $[6,16]$. The thicker film, D after $130 \mathrm{~h}$, exhibits a higher FWHM but still lower than values reported elsewhere [17]. This behavior could be explained by the impact of such thickness. In fact, when thick and highly doped films are obtained, the Fano effect is more evident to observe. In fact, when the analysis is performed on thin film, the influence of the substrate is still present and the FWHM is probably less well evaluated. Thus, for the thicker film at high doping level the Fano effect induces some widening of the diamond peak as clearly shown in previous work [18].

In order to have a more accurate estimation of the doping level, SIMS analysis was performed and the results are reported on Fig.4. It is well known that oxygen addition decreases the boron doping efficiency [7] and that the methane addition increases it [19]. For the present study, the simultaneous increase of both parameters leads to a stable boron doping efficiency with a boron concentration close to $7 \times 1019 \mathrm{at} / \mathrm{cm} 3$ as soon as oxygen concentration is higher than $0.5 \%$. For the lowest oxygen concentration, the higher doping level of $2 \times 1020$ at $/ \mathrm{cm} 3$ could be explained by a decrease of the diamond quality which could be more favorable to boron incorporation. This result is contradictory to what has already been observed in the literature but in the mentioned work, boron concentration in the gas phase was much lower than in our case which could considerably change plasma chemistry and boron incorporation phenomena.

In order to further increase the boron concentration in the diamond films, two additional layers, E and F, were deposited using $10 \%$ of methane, $2 \%$ of oxygen and $20,000 \mathrm{ppm}$ and $30,000 \mathrm{ppm}$ of $(\mathrm{B} / \mathrm{C})_{\text {gas }}$, respectively. The surface morphology of these films was observed by laser confocal microscope and is reported in Fig.5. At 20,000 ppm of (B/C) gas it can be seen that some etch pits begin to appear at the surface and, at higher diborane concentration in the gas mixture, the etch pits density strongly increases as it can be observed on sample F.

Such phenomenon was already observed [20]. Up to now, the boron doping mechanism is not well understood. Several proposals were done concerning the species responsible for doping [6,21]. Especially, high boron concentration in the gas mixture would lead to a strong reduction of the $\mathrm{CH} 3$ radical responsible for diamond growth, even leading to diamond etching. Further study involving modeling and plasma diagnostics are required in order to have a better 
understanding of such phenomena. These two samples were analyzed by SIMS and the boron concentrations are $1.5 \times 10^{20} \mathrm{at} / \mathrm{cm}^{3}$ and $2.7 \times 10^{20} \mathrm{at} / \mathrm{cm}^{3}$ for sample $\mathrm{E}$ and $\mathrm{F}$, respectively. Thus, higher concentration of boron can be reached by increasing the $(B / C)$ gas ratio, even if the growth conditions must be still improved to limit etching phenomena at high diborane concentration.

For the thicker deposited films (Sample D), the enlargement of the grown layer regarding the initial substrate size was estimated. As the grown layer is heavily boron doped and totally dark, it was not possible to measure it starting from optical images. The only way to proceed was to use a $\mathrm{H}_{2} / \mathrm{O}_{2}$ plasma etching during $10 \mathrm{~min}$ at $2700 \mathrm{~W}$ and $170 \mathrm{mbar}$. As we can see in Fig.6, this plasma etching revealed the substrate footprint showing that an enlargement of $1 \mathrm{~mm}$ has been obtained after the growth of $480 \mu \mathrm{m}$ thickness. It corresponds to an increase of the top surface from $10 \mathrm{~mm}^{2}$ to $18 \mathrm{~mm}^{2}$, i.e. roughly a factor of 2 .

\section{Conclusion}

In this paper we presented a new growth protocol of thick and heavily boron doped diamond films on (100) substrates at high microwave power density. The addition of a high amount of oxygen in the gas phase allowed combining for the first time, using a CVD technique, the growth of large, thick and high quality heavily boron doped single crystal diamond. After the growth of $500 \mu \mathrm{m}$-thick layers, the obtained area was roughly two times larger than that of the initial substrate with a boron concentration close to $2 \times 1020 \mathrm{at} / \mathrm{cm} 3$. This concentration can be still increased by increasing boron concentration but the surface morphology exhibits etch pits which should be removed by a polishing step. Further study involving modeling and plasma diagnostics are re- quired in order to have a better understanding of such phenomena and still improve our growth process.

\section{Acknowledgments}

This work was financially supported by ANR through the French national project MoVeToDiam Nº: ANR-17-CE05-0019-02.

\section{References}

[1] J. Isberg, J. Hammersberg, D.J. Twitchen, A.J. Whitehead, Single crystal diamond for electronic applications, Diam. Relat. Mat. 13 (2004) 320-324.

[2] H. Umezawa, Y. Kato, S. Shikata, $1 \Omega$ on-resistance diamond vertical-Schottky barrier diode operated at $250{ }^{\circ} \mathrm{C}$, Appl. Phys. Express 6 (2013) 011302.

[3] P.-N. Volpe, P. Muret, J. Pernot, F. Omnès, T. Teraji, F. Jomard, D. Planson, P. Brosselard, N. Dheilly, B. Vergne, S. Scharnholtz, High breakdown voltage Schottky diodes synthesized on p-type CVD diamond layer, Phys. Stat. Sol. (a) 207 (2010) 2088-2092.

[4] R. Issaoui, J. Achard, F. Silva, A. Tallaire, M.A. Pinault, F. Jomard, A. Tardieu, A. Gicquel, Growth of thick heavily boron-doped diamond single crystals: effect of microwave power density, Appl. Phys. Lett. 97 (2010) 182101.

[5] J. Achard, R. Issaoui, A. Tallaire, F. Silva, J. Barjon, F. Jomard, A. Gicquel, Freestanding CVD boron doped diamond single crystals: a substrate for vertical power electronic devices? Phys. Stat. Sol. (a) 209 (2012) 1651-1658. 
[6] S.A. Bogdanov, A.L. Vikharev, M.N. Drozdov, D.B. Radishev, Synthesis of thick and high-quality homoepitaxial diamond with high boron doping level: oxygen effect, Diam. Relat. Mat. 74 (2017) 59-64.

[7] P.-N. Volpe, J.-C. Arnault, N. Tranchant, G. Chicot, J. Pernot, F. Jomard, P. Bergonzo, Boron incorporation issues in diamond when TMB is used as precursor: toward extreme doping levels, Diam. Relat. Mat. 22 (2012) 136-141.

[8] H. Umezawa, S.-i. Shikata, T. Funaki, Diamond Schottky barrier diode for hightemperature, high-power, and fast switching applications, J. J. Appl. Phys. 53 (2014) 05 FP06.

[9] S. Koné, H. Schneider, K. Isoird, F. Thion, J. Achard, R. Issaoui, S. Msolli, J. Alexis, An assessment of contact metallization for high power and high temperature dia- mond Schottky devices, Diam. Relat. Mat. 27-28 (2012) 23-28.

[10] A. Tallaire, J. Achard, F. Silva, R.S. Sussmann, A. Gicquel, E. Rzepka, Oxygen plasma pre-treatments for high quality homoepitaxial CVD diamond deposition, Phys. Stat. Sol. (a) 201 (2004) 2419-2424.

[11] J. Achard, F. Silva, R. Issaoui, O. Brinza, A. Tallaire, H. Schneider, K. Isoird, H. Ding, S. Kone, M.A. Pinault, F. Jomard, A. Gicquel, Thick boron doped diamond single crystals for high power electronics, Diam. Relat. Mater. 20 (2011) 145-152.

[12] R. Issaoui, J. Achard, A. Tallaire, F. Silva, A. Gicquel, R. Bisaro, B. Servet, G. Garry, J. Barjon, Evaluation of freestanding boron-doped diamond grown by chemical vapour deposition as substrates for vertical power electronic devices, Appl. Phys. Lett. 100 (2012) 122109.

[13] J.E. Butler, R.L. Woodin, Thin film diamond growth mechanisms, Philosophical Transactions of the Royal Society of London, Series A: Physical Sciences and Engineering 342 (1993) 209-224.

[14] V.D. Blank, V.N. Denisov, A.N. Kirichenko, M.S. Kuznetsov, B.N. Mavrin, S.A. Nosukhin, S.A. Terentiev, Raman scattering by defect-induced excitations in boron-doped diamond single crystals, Diam. Relat. Mat. 17 (2008) 1840-1843.

[15] F. Pruvost, A. Deneuville, Analysis of the Fano in diamond, Diam. Relat. Mat. 10 (2001) 531-535.

[16] R. Issaoui, Growth of thick boron doped single crystal diamond by MPAVCD to be used as P+ diamond substrate, 2011, pp. 1 vol. (180 p.).

[17] G. Faggio, G. Messina, S. Santangelo, G. Prestopino, I. Ciancaglioni, M. Marinelli, Raman scattering in boron-doped single-crystal diamond used to fabricate Schottky diode detectors, J. Quant. Spectrosc. Radiat. Transf. 113 (2012) 2476-2481.

[18] A. Boussadi, A. Tallaire, O. Brinza, M.A. Pinault-Thaury, J. Achard, Thick heavily boron doped CVD diamond films homoepitaxially grown on (111)-oriented sub- strates, Diam. Relat. Mat. 79 (2017) 108-111.

[19] M. Tsigkourakos, T. Hantschel, Z. Xu, B. Douhard, J. Meersschaut, Y. Zou, J. Larsson, M. Boman, W. Vandervorst, Suppression of boron incorporation at the early growth phases of boron-doped diamond thin films, Phys. Stat. Sol. (a) 212 (2015) 2595-2599.

[20] A. Fiori, T. Teraji, Plasma etching phenomena in heavily boron-doped diamond growth, Diam. Relat. Mat. 76 (2017) 38-43.

[21] Y.A. Mankelevich, M.N.R. Ashfold, D.W. Comerford, J. Ma, J.C. Richley, Boron doping: $\mathrm{B} / \mathrm{H} / \mathrm{C} / \mathrm{O}$ gas-phase chemistry; $\mathrm{H}$ atom density dependences on pressure and wire temperature; puzzles regarding the gas-surface mechanism, Thin Solid Films 519 (2011) 4421-4425. 
Table 1: Summary of the growth parameters

\begin{tabular}{cccccccc}
$\begin{array}{c}\text { Samples } \\
\text { name }\end{array}$ & $\begin{array}{c}(\mathrm{B} / \mathrm{C})_{\text {gas }} \\
(\mathrm{ppm})\end{array}$ & $\mathrm{O}_{2} \%$ & $\mathrm{CH}_{4} \%$ & $\begin{array}{c}\text { Duration } \\
(\mathrm{h})\end{array}$ & $\begin{array}{c}\text { Thickness as } \\
\text { obtained by } \\
\text { mass loss }(\mu \mathrm{m})\end{array}$ & $\begin{array}{c}\text { Thickness as } \\
\text { obtained by } \\
\text { caliper }(\mu \mathrm{m})\end{array}$ & $\begin{array}{c}\text { Mean thickness } \\
(\mu \mathrm{m})\end{array}$ \\
\hline $\mathrm{A}$ & 15,000 & 0.25 & 5 & 24 & 25 & 27 & 26 \\
$\mathrm{~B}$ & & 0.5 & 6 & 24 & 37 & 37 & 37 \\
$\mathrm{C}$ & & 1 & 7 & 24 & 68 & 71 & 69 \\
$\mathrm{D}$ & & 2 & 10 & 130 & 485 & 475 & 480 \\
$\mathrm{E}$ & 20,000 & & & 24 & 99 & 101 & 100 \\
$\mathrm{~F}$ & 30,000 & & & 24 & Not measurable & Not measurable & Not measurable
\end{tabular}

Table 2: The FWHM as measured by Raman spectroscopy for the sample A, B, C and D.

\begin{tabular}{lc} 
Samples & FWHM $\left(\mathrm{cm}^{-1}\right)$ \\
\hline A & 4.4 \\
B & 4.4 \\
C & 3.9 \\
D after 24h & 5.8 \\
D after 96 & 7.4
\end{tabular}

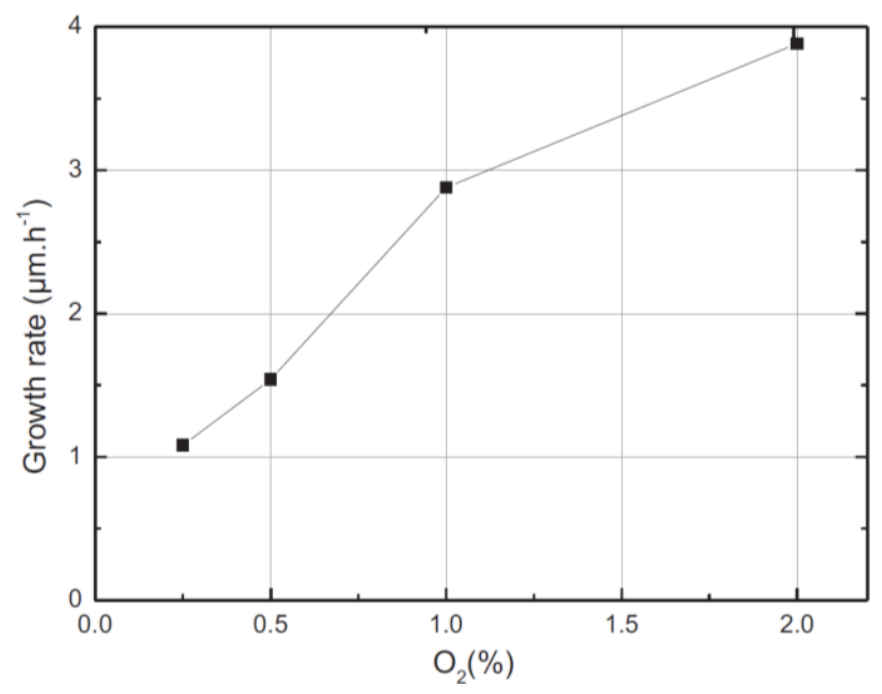

Fig. 1. Evolution of the growth rate with the oxygen content. 


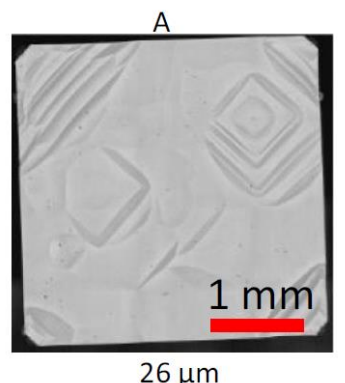

$26 \mu \mathrm{m}$

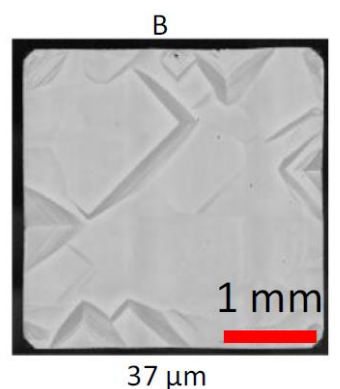

$37 \mu \mathrm{m}$

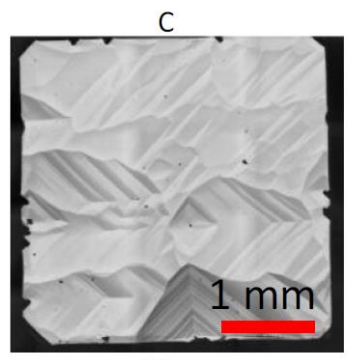

$69 \mu \mathrm{m}$

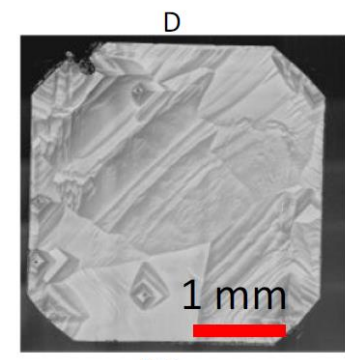

$480 \mu \mathrm{m}$

Fig. 2. Laser confocal microscope images of deposited layers. Samples A to D are presented respectively from the left to the right. The thickness of each sample is reminded below each image.
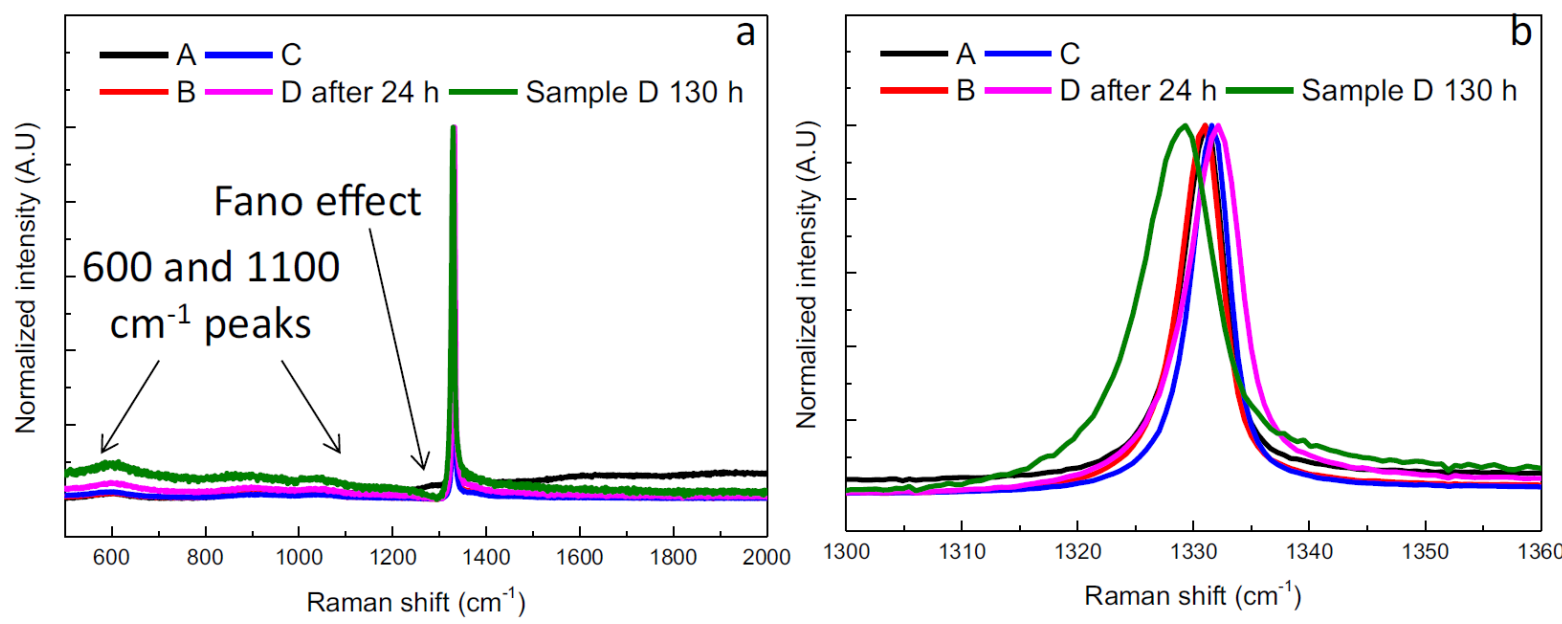

Fig. 3. a) Raman spectra of the samples A, B, C and D. b) Raman spectra of samples A, B, C and $\mathrm{D}$ focused on the day diamond peak.

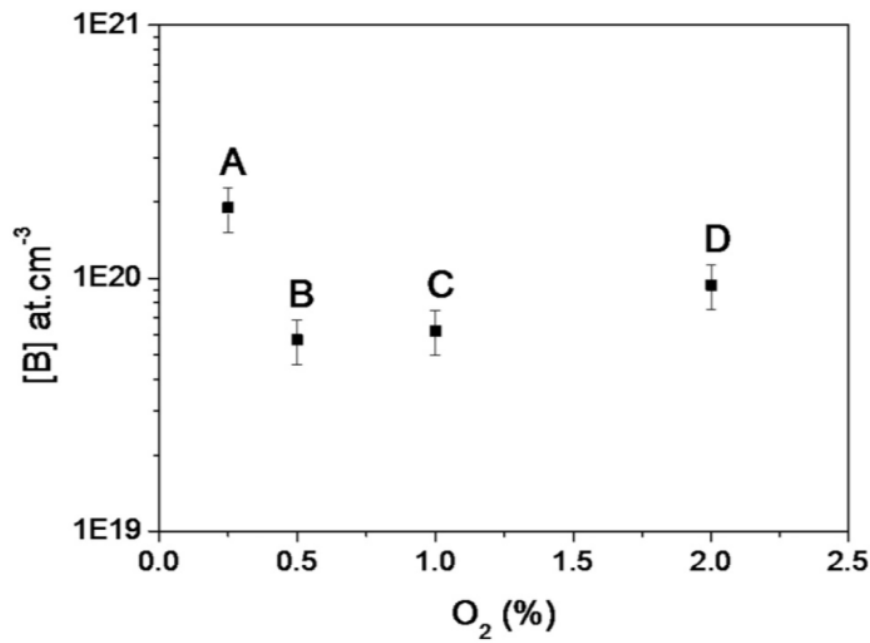

Fig. 4. Evolution of the boron concentration measured by SIMS with the oxygen content $\%$. 



Fig. 5. Laser confocal microscope images of sample E (left) and sample F (right), achieved with $20,000 \mathrm{ppm}$ and $30,000 \mathrm{ppm}$ of $(\mathrm{B} / \mathrm{C})_{\text {gas }}$, respectively.
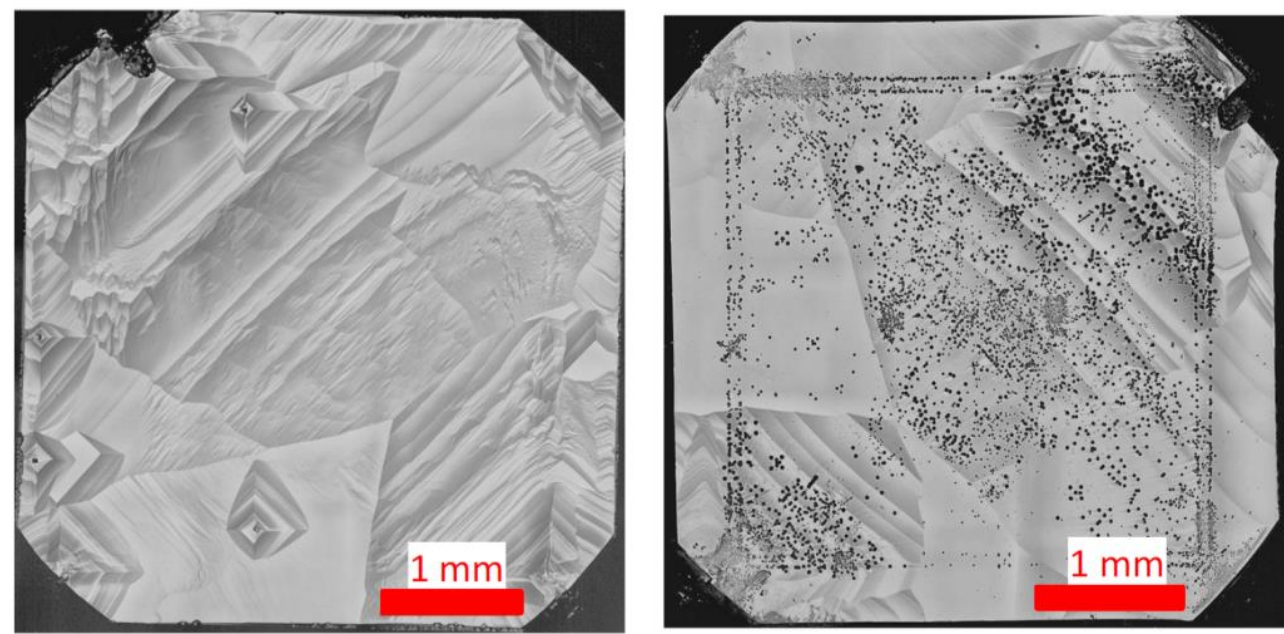

Fig. 6. Confocal laser microscope images of sample D before (left) and after (right) $\mathrm{H}_{2} / \mathrm{O}_{2}$ etching to reveal the substrate footprint. 